\title{
Governments little helper: EU-Politikkoordinierung in der Alterssicherung
}

Zunehmend versucht die Europäische Union (EU) ihren Einfluss auf die Reformen der Alterssicherungssysteme zu mehren. Dabei bleiben die Relevanz und das Potenzial der Offenen Methode der Koordinierung bislang uneindeutig. Der Beitrag beschreibt am Beispiel von Rentenreformen in Frankreich, wie diese sogenannte soft governance im nationalen Reformdiskurs unterstützend genutzt wird.

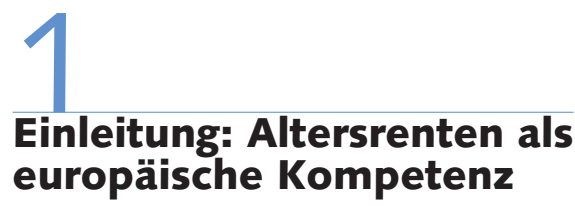

„Die Verantwortung für Pensionen und Renten liegt bei den Mitgliedstaaten", deren Vorrechte oder die Rolle der Sozialpartner in diesem Bereich werden nicht infrage gestellt, argumentiert die Europäische Kommission schon in den einleitenden Sätzen ihres im Juli 2010 veröffentlichten Grünbuchs für „angemessene, nachhaltige und sichere europäische Pensions- und Rentensysteme“ (Europäische Kommission 2010, S. 2). Sozialpolitik ist und bleibt nationalstaatliche Kompetenz in der Europäischen Union (EU), so die über Jahrzehnte gültige Formel. Doch die ökonomische Integration des Kontinents fordert ihren Tribut. Ihre beiden größten Projekte - der gemeinsame Binnenmarkt und die Wirtschaftsund Währungsunion (WWU) - reichen längst in die Ausgestaltung der einzelnen Wohlfahrtsstaaten hinein und erfordern Anpassungen bis hin zu Pfadveränderungen und Systemwechseln. In Ermangelung europäischer Regulierungskompetenz im Bereich der Sozialsysteme wurden mit der Lissabonstrategie im Jahr 2000 Anstrengungen zur europaweiten Koordinierung des Sozialschutzes unternommen. Aufbauend auf der Europäischen Beschäftigungsstrategie (EBS) wurde die Offene Methode der Koordinierung (OMK) in den Sektoren Soziale Eingliederung, Altersrenten und Gesundheit/Langzeitpflege gestartet.

Die OMK stellt sich als vierstufiger rekursiver Prozess dar, bestehend aus:

- der Festlegung gemeinsamer europäischer Leitlinien und Ziele,

- der Formulierung quantitativer und qualitativer Indikatoren und Benchmarks als Messgrößen,
- der Umsetzung der europäischen Leitlinien in nationale Politikvorhaben sowie - der regelmäßigen Überwachung, Bewertung und gegenseitigen Prüfung im Rahmen eines ausgefeilten Berichtswesens.

Erzielt werden soll durch die Interaktion zwischen nationaler und europäischer Ebene im Rahmen von Peer Reviews und der Identifizierung von Best-PracticeBeispielen ein wechselseitiger Lernprozess unter den Mitgliedstaaten.

$\mathrm{Zu}$ den Zielen schreibt der Rat der Europäischen Union (2006, S. 9): Einzelziele der OMK im Rentenbereich (OMK/R) sind „angemessene und tragfähige Rentensysteme durch Gewährleistung

(1) eines angemessenen Ruhestandseinkommens für alle und des Zugangs zu Renten, die es den Menschen erlauben, ihren Lebensstandard nach dem Ausscheiden aus dem Erwerbsleben in einem vernünftigen Rahmen aufrecht zu erhalten, im Geiste der Solidarität und Fairness zwischen den und innerhalb der Generationen;

(2) der finanziellen Nachhaltigkeit der öffentlichen und privaten Rentensysteme, unter Berücksichtigung des Drucks auf die öffentlichen Finanzen und der Bevölkerungsalterung, im Kontext der dreigleisigen Strategie zur Bewältigung der Haushaltsaspekte der Alterung, insbesondere durch Förderung von längerem Erwerbsleben und aktivem Altern, Ausgleich von Beiträgen und Leistungen in angemessener und sozial ausgewogener Weise, Förderung der Bezahlbarkeit und Sicherheit kapitalgestützter und privater Systeme;

(3) von Rentensystemen, die transparent und an die Bedürfnisse und Erwartungen von Frauen und Männern und die Erfordernisse der modernen Gesellschaft, die demografische Entwicklung und den
Strukturwandel angepasst sind; einer Versorgung der Menschen mit den Informationen, die sie für die Planung ihres Ruhestands benötigen, und einer Durchführung von Reformen auf der Grundlage eines möglichst umfassenden Konsensus.“ (Hervorhebungen des Autors)

Der Umweg über „weiche“ Steuerungsformen als genereller Trend der EU-Sozialpolitik in den Bereich der freiwilligen, rechtlich unverbindlichen Koordinierung (Leiber/Schäfer 2008) zeugt einerseits vom Defizit der marktkorrigierenden gegenüber der marktschaffenden Integration (Höpner/Schäfer 2010). Andererseits ist der Versuch zur Koordinierung das Eingeständnis eines zunehmenden Erfordernisses zur Abstimmung der mitgliedstaatlichen Politiken im Integrationsraum. Gerade die Organisation des finanzintensivsten aller Sozialsektoren, der Alterssicherung, kann durch ihren erheblichen Anteil an den öffentlichen Finanzen zu Externalitäten für andere Mitgliedstaaten führen. Im europäischen System von Wettbewerbsstaaten ist die Höhe der Sozialausgaben zunehmend zu einem entscheidenden Standortfaktor geworden (Busch 2009).

Doch stellt die OMK ein einflussreiches Werkzeug im Instrumentenkasten der politischen Akteure dar, um die grundlegende Asymmetrie der EU zwischen negativer und positiver Integration (Scharpf 1999, S. 53) zu überwinden? Bislang ist unklar, ob sich durch die OMK eine „new mode of governance“ (Hodson/Maher 2001)

\footnotetext{
Björn Hacker, Dr., Politikwissenschaftler und Referent in der Friedrich-EbertStiftung (FES). Arbeitsschwerpunkte: Wohlfahrtsstaatsreformen, Europäische Integrationsforschung, internationale Wirtschaftspolitik. e-mail: bjoern.hacker@fes.de
} 
durchsetzt, also eine Regierungsform, die relativ wenig Gebrauch macht von Mitteln der hierarchischen Steuerung, sondern auf Handlungskoordination zwischen staatlichen und nicht-staatlichen Akteuren beruht. Gelingt es der OMK, durch ein systematisiertes "gegenseitiges Lernen“ eine europäische Synthese positiver Reformerfahrungen der Mitgliedstaaten zu befördern oder befeuert sie umgekehrt eine „wettbewerbsgetriebene Harmonisierung über Umwege" (Urban 2003, S. 50; Platzer 2009, S. 116)?

In diesem Beitrag wird anhand des Länderbeispiels Frankreich, wo im letzten Jahrzehnt besonders viele umfassende Reformschritte stattfanden, analysiert, inwieweit die OMK im Bereich der Altersrenten $(\mathrm{OMK} / \mathrm{R})$ das Potenzial für eine „new mode of governance“ besitzt, mit deren Einfluss die soziale Dimension des europäischen Integrationsprozesses gestärkt werden könnte.

\section{Methodik: Messung des Politiktransfers}

Um den Einfluss der OMK zu messen, hat sich in den Sozialwissenschaften bislang noch kein einheitliches Analyseverfahren durchgesetzt. Im Gegenteil zeichnet sich der Zugang durch eine große Varianz unterschiedlicher theoretischer und methodischer Ansätze aus (Hartlapp 2006; Citi/Rhodes 2007; De la Porte 2010). Dabei liegt der Schwerpunkt der GovernanceForschung auf Verfahrensaspekten der OMK. So wird mehrheitlich untersucht, inwieweit sich Lerneffekte durch die Koordinierung einstellen, wie ausgeprägt sich die Partizipation gesellschaftlicher Akteure gestaltet und wie es um Transparenz und Legitimität des Verfahrens bestellt ist. Methodisch wird oft die Form von Interviews mit Ministerial- und Kommissionsbeamten und weiteren Akteuren, wie etwa Sozialpartnern und Nichtregierungsorganisationen, gewählt. Im Hintergrund dieser Untersuchungen steht fast immer der Anspruch, Effektivität und Einfluss der Koordinierungsmethode festzustellen. Doch während diese Annäherung fast ausschließlich über Verfahrensregularien, verfasste Dokumente und die an ihrer Erstellung beteiligten Personen kreist, wird der Hauptort des politischen Geschehens im demokratischen Staat - das Parlament - weitgehend außer Acht gelassen. Die Argumentationskette hierfür ist denkbar einfach: Die Parlamente haben in der OMK keine offizielle Funktion, ergo lässt sich der Wirkungsgrad dieser Methode auch nicht im Parlament messen. Doch ob und inwieweit ein Politiktransfer von der supranationalen Koordinierung auf die nationale Ebene stattfindet, misst sich in der nationalen Politikarena im Zentrum des Reformgeschehens. Dies ist im demokratischen Staat der parlamentarische Diskurs. Hier kommen alle für den jeweiligen Politikbereich relevanten nationalen Akteure zu Beratungen zusammen, und wenn die OMK einen Einfluss hat, sollte sich dieser in expliziten Referenzen oder implizit in der Verwendung ihrer Zielkriterien als Bestandteil der im Parlament verhandelten Gesetzesvorhaben zeigen. Mehr noch: Der Fokus der OMK-Forschung auf die Nutzung der OMK durch die nationalen Regierungsapparate lässt außer Acht, dass die Parlamentarier das letzte Wort über politische Vorschläge der jeweiligen Regierung sprechen und deren Pläne abändern und verwerfen können (De Ruiter 2010, S. 876).

Die Feststellung des Grads an Politiktransfer (Dolowitz/Marsh 2000) von der Koordinierungsmethode auf EU-Ebene in die nationalen Politikarenen ist unabdingbar, um den Einfluss der OMK zur Stärkung der sozialen Dimension des Integrationsprozesses messen zu können. Unabhängig davon, nach welchem der drei dominierenden Erklärungsansätze der Governance-Forschung - Politikdiffusion, Politiklernen oder Wettbewerb (vgl. Radaelli 2003; Holzinger et al. 2007; Benz 2007) ein Politiktransfer festgestellt werden soll, ist nach Reimut Zohlnhöfer und Tobias Ostheim bei der Messung des Einflusses der OMK auf die nationalen Politiken ein analytischer Dreischritt vorzunehmen. Dabei wird erstens geprüft, ob eine Kongruenz zwischen den Zielen der OMK und der nationalen Reformpolitik festgestellt werden kann. Dies kann mittels einer inhaltsanalytischen Gegenüberstellung der Nationalen Reformpläne mit den bewertenden Gemeinsamen Berichten von Europäischer Kommission und Rat erfolgen. Die Übereinstimmung zwischen den europäischen Zielsetzungen und der im jeweiligen Bereich betriebenen Politik eines Mitgliedstaats ist die „conditio sine qua non" für die Existenz eines Politiktransfers (Zohlnhöfer/Ostheim 2007, S. 333). Doch dies sagt noch nichts aus über die faktische politische Verwendung der OMK. Daher muss zweitens untersucht werden, in welchem Ausmaß in den parlamentarischen Beratungen politischer Reformen Referenzen zur OMK vorhanden sind. Hierzu ist diskursanalytisch zu prüfen, ob und wie am Gesetzgebungsverfahren beteiligte Akteure die OMK und ihre Ziele in ihre politische Strategie einbinden. Über Kodierungen und Klassifizierungen aus dem umfassenden Textkorpus aus Protokollen von Ausschusssitzungen, Anhörungen, Gesetzesbegründungen und Stellungnahmen können regelmäßig auftretende Phänomenstrukturen, Akteurskonstellationen und Subjektpositionen identifiziert werden (vgl. Diaz-Bone 2006; Keller 2007). Da aber auch Kongruenzen und Referenzen zusammengenommen noch keine Einflussnahme beweisen, wird drittens festgehalten, an welchen Stellen nicht von einem Politiktransfer auszugehen ist, da spezifische Politiken auf alternative Erklärungsfaktoren zurückzuführen sind (Evans/Davies 1999, S. 382; Zohlnhöfer/ Ostheim 2007, S. 334). Dies ist möglich, indem die im Diskurs zurate gezogenen, die Reform vorbereitenden und begleitenden Dokumente als Alternativen geprüft und ihrerseits auf Verbindungen zur OMK untersucht werden.

Die Messergebnisse einer dergestalt durchgeführten Analyse von Politiktransfer durch offene Politikkoordinierung auf europäischer Ebene sind nach Zohlnhöfer/ Ostheim (2007, S. 334) in zwei möglichen Effekten darstellbar - einem Lenkungsund einem Verstärkereffekt. Bei einem Lenkungseffekt wird die nationale Politik maßgeblich von der OMK beeinflusst; bei ihrer Abwesenheit ist von einem anderen Verlauf der Entscheidungsfindung und des Ergebnisses auszugehen. Dieser lenkende Effekt ist nur möglich, wenn bei gegebener Kongruenz viele Referenzen zur OMK und wenige alternative Erklärungsfaktoren vorhanden sind. Ein Verstärker- oder Katalyseeffekt unterstützt die nationalstaatlichen Akteure zur Verfolgung einer bestimmten, durch sie selbst gewählten Reformrichtung. Diese Wahl hätten die Akteure auch bei Abwesenheit der OMK getroffen; die Akteure nutzen die europäische Ebene in einem strategischen Sinne, um ihre Argumente zu untermauern und die Durchsetzung ihrer Politik zu erleichtern. Ein katalysierender Effekt stellt sich bei gegebener Kongruenz durch eine Viel- 
zahl an Referenzen wie auch alternativen Erklärungsfaktoren ein. Zu ergänzen ist ein dritter möglicher Effekt einer neutralen Wirkung der OMK (vgl. Chalmers/Lodge 2003) auf die nationale Politikgestaltung. Liegt ein neutraler Effekt vor, sind bei gegebenen Kongruenzen nur wenige Referenzen vorhanden, es steht jedoch eine Vielzahl an alternativen Erklärungsfaktoren bereit (vgl. Hacker 2010, S. 102ff.).

\section{Relevanz der OMK in der französischen Reformpolitik}

Von Mitte der 1980er Jahre bis 2002 verfolgten die französischen Regierungen eine Politik der Nicht-Entscheidung bezüglich einer umfassenden Reform der Alterssicherung. Von drei geplanten größeren Reformschritten scheiterten zwei durch Rücknahme der geplanten Maßnahmen infolge massiver sozialer Proteste (Plan Juppé) oder eines Regierungswechsels (Loi Thomas). Als einzige erfolgreiche Reform des Rentensystems konnte lange Zeit ausschließlich die Balladur-Reform des Jahres 1993 gelten, deren Reichweite zur Sicherung der finanziellen Stabilität des Systems jedoch begrenzt war (vgl. Schludi 2005, S. 212).

2003 wagte die mit einer bequemen Mehrheit in beiden Kammern des Parlaments ausgestattete Mitte-Rechts-Regierung von Premierminister Jean-Pierre Raffarin eine umfassende Rentenreform. Mit der nach dem damaligen Sozial- und Arbeitsminister benannten Fillon-Reform fand nun die Angleichung des Beamtensystems an das Régime général der in der Privatwirtschaft Beschäftigten statt; die notwendige Beitragsdauer für den Erhalt einer abschlagsfreien Rente wurde (schrittweise bis 2008) auf 40 Jahre erhöht. Zudem wurde für die Versicherten beider Systeme eine weitere Stärkung der Beitrags-Leistungs-Beziehung durch Ausweitung der Qualifikationszeit, Beschränkung der Frühverrentungsmöglichkeiten und Implementierung eines Bonus/Malus-Systems für den Austritt aus dem Erwerbsleben vor bzw. nach dem gesetzlichen Rentenalter, das bei 60 Jahren belassen wurde, auf den Weg gebracht. Hinzu kommt die Institutionalisierung der privaten kapitalgedeckten Altersvorsorge im Rahmen des Plan dépargne retraite populaire und des Plan dépargne retraite collective. Mit der so geschaffenen, wachsenden Bedeutung der privaten Altersvorsorge wurde eine Ersatzfunktion für die durch die Reformen kontinuierlich sinkende Ersatzrate geschaffen (Palier 2003). Mittels präsidialer Dekrete und nur unter Konsultierung der Assemblée Nationale und des Sénats trat zum 1. Juli 2008 eine Reform der Régimes Spéciaux der großen Staatsbetriebe (etwa Electricité de France (EDF), Sociéte nationale des chemins de fer français (SNCF), Régie autonome des transports Parisiens (RATP)) in Kraft. Diese eigenständigen Rentensysteme wurden an die 2003 verabschiedeten Konditionen des Beamtensystems angeglichen. Damit lag eine weitgehende Harmonisierung des kompliziert verzweigten französischen Rentensystems vor. Mit der Fillon-Reform wurde ein sog. „Rendez-vous 2008“ vereinbart. Zu diesem Zeitpunkt sollte die Wirksamkeit der beschlossenen Maßnahmen evaluiert und unter Umständen ergänzt werden. Weiterführende Reformmaßnahmen wurden daraufhin im Rahmen des jährlichen Gesetzes zur Finanzierung der Sozialversicherung (Projet de Loi de Financement de la Sécurité Sociale, PLFSS 2009) im November 2008 vom Parlament verabschiedet. Bestätigt wurde der bereits 2003 anvisierte schrittweise Übergang von $40 \mathrm{zu} 41$ Versicherungsjahren für den Erhalt einer abschlagsfreien Rente für alle Versicherten. Der Zugang zur 2003 neu beschlossenen Regelung für den Ruhestand vor Erreichen des gesetzlichen Rentenalters bei besonders langjährig Versicherten wurde erschwert. Dagegen wurde die Mindestrente für Empfänger des Mindestlohns SMIC in Höhe von $85 \%$ bis 2012 fortgeschrieben. Besondere Aufmerksamkeit wurde der Steigerung der Beschäftigungsmöglichkeiten älterer Arbeitnehmer gewidmet, etwa durch die vollständige Freiheit zur Kumulierung von Rente und Erwerbseinkommen für Versicherte oder die Erhöhung des Rentenzuschlags bei Verbleib in der Beschäftigung über das gesetzliche Rentenalter hinaus. Durch die akkumulierte Wirkung der Rentenreformen von 1993, 2003 und 2008 entwickelte sich die Alterssicherung in Frankreich von einem leistungs- (defined benefit) zu einem beitragsdefinierten (defined contribution) System weiter, ${ }^{1}$ indem der versicherungsmathematischen Aktuarität und der privaten Altersvorsorge sowie der bedarfsabhängigen Mindestsicherung zunehmend Raum gegeben wurde (vgl. Hacker 2010, S. 152ff.).

\subsection{KONGRUENZEN MIT DER NATIONALEN REFORMPOLITIK}

Die Betrachtung der drei Koordinierungszyklen sowie der zwischenzeitlichen Berichterstattung des OMK-Prozesses zwischen 2002 und 2009 zeigt Frankreich als kooperativen Partner der EU. In ihrem Nationalen Strategiebericht (NSB) zur Alterssicherung von 2002 diskutiert die französische Regierung sehr offen und durchaus selbstkritisch die Unzulänglichkeiten und den Reformbedarf des französischen Rentensystems, orientiert an den Zielvorgaben der OMK/R: Auf das baldige Erreichen des Rentenalters der ersten Nachkriegsgeneration (Baby-Boomer) sei das Rentensystem Frankreichs nur bedingt vorbereitet. Die Alterung der Gesellschaft könne dabei nur partiell von einer steigenden Beschäftigungsquote kompensiert werden. Reformen zur Verbesserung der Relation von Beitragszahlern und Rentenbeziehern seien daher unumgänglich (Gouvernement de la République Française 2002, S. 7f.). Die größte Herausforderung liegt laut Kommission und Rat in der Sicherung der finanziellen Nachhaltigkeit (Europäische Kommission 2003, S. 133f.).

Im zweiten NSB von 2005 kann die Regierung von Premierminister Dominique de Villepin auf die 2003 verabschiedete Fillon-Reform zurückblicken. Davon wird ausführlich Gebrauch gemacht. Zu jedem der OMK-Einzelziele findet sich hier eine Entsprechung in der jüngsten nationalen Rentenpolitik. Die selbstkritische Distanz des ersten NSB wird ersetzt durch die ständige Betonung, dass die Reform ausgewogen und ihr mittelfristiges Ergebnis vollkommen im Einklang („pleinement cohérente") sei mit den Zielen der OMK/R (Gouvernement de la République Française 2005, S. 12). Entsprechend lobt der sog. Synthesebericht von Kommission und Rat zur OMK/R im Jahr 2006 Frankreich für die 2003 durchgeführte Rentenreform. Doch nach wie vor sei Frankreich von einem hohen Finanzdruck infolge der Alterung der Gesellschaft und einer hohen öffentlichen Verschuldung betroffen.

1 Nicht betrachtet wird im Folgenden die letzte Reform Ende 2010, die mit der Erhöhung des Renteneintrittsalters jedoch ebenfalls dem beschriebenen Trend folgt. 
Schon heute überstiegen die Rentenausgaben als Anteil des Bruttoinlandsprodukt (BIP) mit $13 \%$ das europäische Mittelmaß, allerdings konnte der Ausgabenanstieg durch die Reform gedämpft werden. Das Land gehöre aber zur Gruppe jener Mitgliedstaaten, in denen die private Altersvorsorge kaum eine Rolle spiele. Besorgt äußern sich Kommission und Rat zur weiterhin sehr geringen Erwerbsquote der älteren Arbeitnehmer. 2004 erreichte Frankreich bei der Beschäftigung der 55bis 64-Jährigen nur einen prozentualen Anteil von 37,3\%. Hinsichtlich des Rahmenziels der Angemessenheit der Renten wird Frankreich bescheinigt, über derart positive Werte von niedrigen Armutsraten und eines hohen Einkommensniveaus für ältere Menschen zu verfügen, dass zurzeit keinerlei Handlungsbedarf in diesem Bereich gesehen werde. Da durch die Reform von 2003 aber das Nettorentenniveau von einer heute 80 \%igen Ersatzrate (bei einem Durchschnittsverdiener mit Renteneintritt mit 65 und nach 40 Beitragsjahren) auf $63 \%$ im Jahr 2050 absinken werde, solle die weitere Entwicklung genau beobachtet werden. Mit der Fillon-Reform habe Frankreich auch maßgeblich zur Modernisierung der Alterssicherung beigetragen, stellt der europäische Synthesebericht fest. Hervorgehoben wird die Verringerung der Ungleichheiten zwischen den verschiedenen Einzelsystemen (Europäische Kommission 2006).

Der NSB 2008, von der Regierung unter Premierminister François Fillon verfasst, stellt relativ nüchtern die Evolution des französischen Systems der Alterssicherung einschließlich der bislang verabschiedeten Reformen dar. Die Gewissheit, mit der im NSB 2005 noch behauptet wurde, die Fillon-Reform von 2003 sichere langfristig die finanzielle Nachhaltigkeit des Rentensystems, weicht einer Analyse der positiven und negativen Effekte dieser Reform (Gouvernement de la République Française 2008). Die weiterhin geringe Beschäftigungsquote älterer Arbeitnehmer bleibt der neuralgische Punkt in der Bewertung von Frankreichs Reformpolitik: Sollte sich bei der schlechten Beschäftigungssituation der 55- bis 64-Jährigen keine Besserung einstellen, sehen Kommission und Rat das Land mit einer doppelten Herausforderung konfrontiert: erstens mit dem projizierten Sinken der Nettoersatzrate in einem unter den EU-Staaten besonders hohen Maße von 79,7 \% im Jahr 2006 auf 62,2 \% im Jahr
2046 und zweitens mit dem Anstieg der öffentlichen Verschuldung, auch durch die Ausgaben im Bereich der Altersrenten, die von 13,1 \% des BIP im Jahr 2007 auf 14,8 \% im Jahr 2050 ansteigen würden. Frankreich sei weit entfernt vom Lissabonziel einer Erwerbsquote in dieser Altersgruppe von mindestens $50 \%$ bis 2010, wie es auf dem Gipfel des Europäischen Rats in Stockholm 2001 vereinbart wurde. Das Gleiche gilt für den Beschluss von Barcelona 2002, das effektive Renteneintrittsalter bis 2010 im Vergleich zu 2001 um fünf Jahre anzuheben. Mit etwa 57,5 Jahren weise Frankreich eines der niedrigsten effektiven Renteneintrittsalter in der EU auf. Die Reform der Régimes Spéciaux werde nur wenig zur Stärkung der finanziellen Nachhaltigkeit des Rentensystems beitragen, da sie nur etwa $2 \%$ der Erwerbstätigen betreffe, jedoch trage die Maßnahme zur gesteigerten Gleichbehandlung der Versicherten und Systemharmonisierung bei (Europäische Kommission 2009, S. 102, 120).

Auch wenn Frankreich kein Musterschüler im Sinne der vollen Erfüllung aller OMK-Ziele ist, sind die Reformanstrengungen doch eindeutig den drei Zielkriterien der Koordinierungsmethode zuzuordnen. Selbst in Bereichen, in denen die Regierung selbst keine Lösungsansätze oder Reformpläne anzubieten hat, verweigert sie sich nicht einer Problembeschreibung. Auch geht sie sehr detailliert auf die von Kommission und Rat in den Gemeinsamen Berichten geäußerten Kritikpunkte ein, indem entsprechende Schwerpunkte im jeweils nächsten NSB gesetzt werden, sodass der Eindruck eines über die Zeit gestreckten Dialogs entsteht (Hacker 2010, S. 267ff.). Daher kann von einer weitgehenden Kongruenz der Rentenreformpolitiken mit den Zielen der OMK ausgegangen werden.

\subsection{REFERENZEN ZUR EUROPÄISCHEN DIMENSION}

Das gleiche europäische Bewusstsein bei der Darstellung und Analyse der nationalen Entwicklungen in der Alterssicherung, das schon die Nationalen Strategieberichte widerspiegeln, drückt sich auch in den parlamentarischen Diskursen aus. Dies gilt in besonderem Maße für die Fillon-Reform, da hier die Regierung Raffarin, vor allem in Person des zuständigen Sozial- und Arbeitsministers François Fillon, bereits in der Reformvorbereitung auf die europä- ische Dimension hinweist. So absolviert er eine medial inszenierte Informationsreise in die Nachbarstaaten Deutschland, Schweden, Finnland und Spanien, um vor Ort in Gesprächen mit Vertretern der Ministerien, den Rentenversicherungsträgern und den Sozialpartnern Eindrücke über die Reformtätigkeiten in den jeweiligen Ländern zu gewinnen. Diese speist der Minister sehr häufig in die parlamentarische Reformdiskussion ein. Dabei ist das zentrale Argument, dass die meisten europäischen Länder bereits umfassende Rentenreformen durchgeführt hätten und Frankreich nun unbedingt nachziehen müsse. Außer Frankreich sei es allen EU-Ländern gelungen, auf die Herausforderungen der demografischen und wirtschaftlichen Entwicklung angemessen zu reagieren und ihre Rentensysteme entsprechend anzupassen (Sénat 2003, S. 21; Sozial- und Arbeitsminister François Fillon, Assemblée Nationale 2003a, S. 314). Daher versucht die Regierungskoalition den Eindruck zu erwecken, dass ganz Europa auf den Spätzünder Frankreich warte und mehr noch: dass nur mittels der von der Regierung eingebrachten Rentenreform die Glaubwürdigkeit Frankreichs, überhaupt reformfähig zu sein, in der EU wiederhergestellt werden könne (Ministre de la fonction publique, de la réforme de l'Etat et de l'aménagement du territoire JeanPaul Delevoye, Union pour un mouvement populaire (UMP), Assemblée Nationale 2003b, S. 6722). Fillon stößt damit eine Tür auf, hinter der sich ein mehrheitlich schlechtes Bild Frankreichs im Vergleich mit seinen europäischen Nachbarstaaten verbirgt. Dieses Bild wird verstärkt durch Verweis auf die im Gemeinsamen Bericht 2003 von der EU angeprangerten schlechten Indikatorenwerte Frankreichs in Bezug auf die Erwerbstätigenquote Älterer, die projizierte Entwicklung der staatlichen Rentenausgaben und das frühe Renteneintrittsalter. Die Regierungsmehrheit nutzt die relative Reformrückständigkeit des eigenen Landes, um den von ihr geplanten Veränderungen in der Alterssicherung mehr Nachdruck zu verleihen. Fast alle Einzelmaßnahmen der FillonReform werden unter anderen Aspekten auch mit den Beispielen aus EU-Ländern begründet, deren Reformbilanz angeblich positiv ist. Dies setzt sich bei den Folgereformen 2008 fort (vgl. Arbeits- und Sozialminister Xavier Bertrand, UMP, Sénat 2007, S. 3478; Senator Dominique 
Leclerc, Vorsitzender des Sozialausschusses UMP, Sénat 2008a , S. 6720).

Die Opposition hat es schwer, diesen Vergleichen etwas entgegenzusetzen. Sie versucht es, indem sie die vom Minister vorgetragenen Daten anzweifelt oder den Reformbedarf durch Nennung von Gegenargumenten (hohe Geburtenrate und Arbeitsproduktivität) negiert (vgl. Abgeordneter Jean-Pierre Brard, Parti communiste français (PCF), Assemblée Nationale 2003c). Nur am Rande benennt sie negative Konsequenzen der Rentenreformen anderer EU-Staaten, vor allem die voraussehbar erheblich sinkenden Ersatzraten (vgl. Abgeordneter André Vallini, Parti socialiste (PS), Assemblée Nationale 2003d, S. 5170). Gerade weil Frankreich im europäischen Vergleich so niedrige Werte des effektiven Renteneintrittsalters und der Erwerbstätigkeit älterer Arbeitnehmer aufweise, sei es scheinheilig, längere Beitragszeiten von den Versicherten zu verlangen, die letztlich nur zu einer Rentenkürzung führen würden (vgl. Senatorin Raymonde Le Texier, PS, Sénat 2008b, S. 7012f.).

Der Koalition aus UMP und Union pour la démocratie française (UDF) gelingt es, die parlamentarische Opposition mit dem Argument des allgemeinen Reformrückstands des Landes vor sich herzutreiben. Denn eine Verbesserung der französischen Indikatoren zur Alterssicherung im europäischen Konzert stellt ein überparteiliches Anliegen dar. Neben ökonomischen Aspekten der Wettbewerbsfähigkeit und der Einhaltung der Kriterien des Stabilitäts- und Wachstumspakts stechen die Erwerbsquote älterer Arbeitnehmer und das effektive Renteneintrittsalter als Reformbegründungen im europäischen $\mathrm{Zu}$ sammenhang hervor. Auch wenn der auf die EU bezogene Diskurs bei der Reform der Régimes Spéciaux und beim PLFSS 2009 insgesamt relativ schwach bleibt, ziehen sich die für Frankreich scheinbar unerfüllbaren Lissabonkriterien der Gipfeltreffen von Stockholm und Barcelona als Referenzen durch die Beratungen dieser beider Reformvorhaben (Hacker 2010, S. 277ff.).

Die niedrige Erwerbsquote Älterer und das relativ frühe Renteneintrittsalter sind zugleich die prioritären Kritikpunkte von Kommission und Rat in den Gemeinsamen Berichten der OMK/R. Dies ist noch keine hinreichende Begründung für eine direkte Wirkung der OMK/R auf die französischen Reformen im Bereich der Alterssicherung, zumal beide Ziele sich auch in der Europäischen Beschäftigungsstrategie wiederfinden. Die Zielkriterien der OMK/R ergänzen jedoch die eigenen Anstrengungen der französischen Politik zur Information in und zum Lernen von anderen EU-Staaten, was sich in den Ausschussarbeiten explizit und in den Plenarberatungen von Assemblée Nationale und Sénat implizit bemerkbar macht.

\subsection{EXISTENZ ALTERNATIVER ERKLÄRUNGSFAKTOREN}

Insgesamt stehen mit Demografie, Finanzdruck in den Rentenkassen, Harmonisierungsbestrebungen und dem so zwar nicht formulierten, faktisch jedoch stattfindenden Projekt eines Umbaus der Alterssicherung von einem leistungsdefinierten $\mathrm{zu}$ einem beitragsdefinierten System für die hier analysierten Parlamentsdiskurse genügend externe Erklärungsfaktoren bereit (vgl. Hacker 2010, S. 288ff.). Zudem existiert eine Vielzahl an Studien und Berichten, auf die sich im Reformdiskurs häufig bezogen wird und die als Reform vorbereitend einzustufen sind. Doch können diese nur schwerlich als „alternativ“ zum Einfluss der OMK/R angesehen werden. Denn im Diskurs weisen auch die zurate gezogenen Unterlagen einen starken Bezug zur europäischen Dimension auf. Die Darstellung und der Vergleich von Rentensystemen und -reformen anderer europäischer Staaten mit dem eigenen Land hat in Frankreich eine Tradition, die hier bis zum „Livre blanc sur les retraites“ von 1991, mit dem die Reformdebatte politisch lanciert wurde, zurückverfolgt werden kann. Dieses Weißbuch zeigt für die Zeit seines Erscheinens eine enorme Weitsicht, wenn es die Verwirklichung der vier Binnenmarktfreiheiten des freien Verkehrs von Gütern, Dienstleistungen, Kapital und Personen als besondere Herausforderung für die bislang national abgeschlossenen Systeme der Alterssicherung bezeichnet. Fast schon unter Vorwegnahme der OMK/R wird ein Systemvergleich der damals zwölf EWGStaaten durchgeführt, der die Organisationsstrukturen, Finanzierungsgrundlagen und Rentenformen beschreibt sowie Vergleiche anstellt zur in der Rentenformel berücksichtigten Beitragsdauer, zum gesetzlichen Renteneintrittsalter und zur Funktionsweise der jährlichen Rentenanpassung. Schon 1991 wird bei diesem Vergleich deutlich, dass Frankreich über eine relativ kurze Qualifikationsperiode und nach Italien über das geringste gesetzliche Rentenalter verfügt (Commissariat général du Plan 1991, S. 207ff.).

Auch der 1999 erschienene Rapport Charpin enthält ein eigenes Kapitel über Rentenreformen in anderen Ländern. Ausgewählt wurden jene Staaten, die ihre Alterssicherung zum damaligen Zeitpunkt bereits maßgeblich verändert hatten oder im Begriff waren, dies zu tun. Dies waren insbesondere die EU-Staaten Italien, Deutschland, Großbritannien und Schweden. Neben der Vorstellung grundlegender Systemkriterien und einzelner Indikatoren, wie Rentenalter, Qualifikationszeit, Abdeckungsrate und Rentenausgaben in Prozent des BIP, werden vor allem die jüngsten Reformschritte und Reformabsichten und ihre möglichen Auswirkungen diskutiert (Charpin 1999, S. 59ff.).

Eingerichtet im Jahr 2000 von der Regierung Lionel Jospin, hat der Conseil d'Orientation des Retraites (COR) in allen seit diesem Zeitpunkt verabschiedeten größeren Reformwerken der Alterssicherung eine tragende Rolle in der Vorbereitung der Maßnahmen übernommen. Von allen externen Faktoren, die im Reformdiskurs genannt werden, sind die Referenzen zu den Studien des COR mit Abstand am zahlreichsten. Mindestens alle zwei Jahre erstellt der COR einen Bericht an Premierminister und Parlament, der die Gesamtsituation der Alterssicherung analysiert und geeignete Maßnahmen zum langfristigen Erhalt des Systems vorschlägt. Mit der Fillon-Reform wurden die Kompetenzen des COR präzisiert. Demnach gehören zu seinen Kernanliegen neben der Wahrung der finanziellen Nachhaltigkeit der Rentensysteme auch die Beachtung eines angemessenen Rentenniveaus, insbesondere durch Beobachtung der Ersatzratenentwicklung sowie die Beteiligung an der Information der Bevölkerung über durchgeführte Reformen und die ihnen zuzuschreibenden Effekte (Loi du 21 août 2003, Article 6). Diese 2003 ergänzte Aufgabenbeschreibung ist mit ihren drei Feldern nahezu deckungsgleich mit den drei Rahmenzielen der OMK/R. In Bezug auf die Orientierung an anderen Ländern enthält der erste COR-Bericht ein eigenes Kapitel, in dem Reformmaßnahmen in europäischen Nachbarstaaten im Hinblick auf eine Adaption in Frankreich diskutiert werden. Dabei wird zunächst die Kompetenz der 
EU im Bereich Alterssicherung erläutert, wobei die Beschäftigungsziele der Lissabonstrategie ebenso erwähnt werden, wie die zur Zeit der Berichtserstellung gerade vorbereitete Implementierung der OMK/R (COR 2001, S. 167).

Auch die Relevanz der OMK/R wird analysiert. Es werden Zweifel hinsichtlich der Durchsetzungskraft der OMK/R, der Richtigkeit der in ihrem Rahmen angestellten Vergleiche angesichts sehr unterschiedlicher Ausgangsbedingungen der EU-Staaten, der hinreichenden Einbindung politischer und sozialer Akteure in den Prozess und der Gleichgewichtigkeit ökonomischer und sozialer Ziele geäußert ${ }^{2}$ (COR 2004, S. 292). Jedoch sieht der COR zugleich die Notwendigkeit einer verstärkten Koordinierung der Alterssicherungspolitiken in Europa. Den politischen Akteuren in Frankreich empfiehlt der COR, trotz der nicht perfekt ausgestalteten $\mathrm{OMK} / \mathrm{R}$ sich für die Entwicklung der Alterssicherungssysteme anderer Länder $\mathrm{zu}$ interessieren und sich von einzelnen Reformen in der eigenen Politik inspirieren zu lassen. Dies gelte besonders für den Bereich der Beschäftigung älterer Arbeitnehmer, in dem Frankreich - wie der COR 2004 erkennt - im europäischen Vergleich besonders schlecht abschneide und u. U. von anderen Staaten lernen könne, wie diese Situation zu verändern sei (COR 2004, S. 332). Die Erwerbsquote älterer Arbeitnehmer bleibt fortan ein Fixpunkt der COR-Berichte, wobei stets auf die europäischen Verpflichtungen im Rahmen der Lissabonstrategie aufmerksam gemacht wird (COR 2004, S. 314ff.; COR 2006, S. 39ff.). Ähnlich den Gemeinsamen Berichten im Rahmen der OMK/R identifiziert auch der vierte Bericht des COR vom Januar 2007 das niedrige effektive Renteneintrittsalter und die zahlreichen Möglichkeiten zur Frühverrentung als hauptsächliche Ursachen für die niedrige Erwerbsquote der 55- bis 64-Jährigen (COR 2007, S. 30ff.).

Die im Rahmen des COR weiter betriebenen eigenen Vergleichsstudien treten somit keineswegs in Konkurrenz zur $\mathrm{OMK} / \mathrm{R}$, denn sie greifen deren Inhalte und Ergebnisse auf und speisen sie so in den politischen Prozess der Rentenreformen ein. Für Frankreich kann daher von einem Ineinandergreifen aus in europäischer und nationaler Regie durchgeführten Evaluierungen des Alterssicherungssystems gesprochen werden.

\section{4 Strategische Nutzung
der OMK als Reform-
Katalysator}

Die europäische Dimension hat in den drei hier untersuchten Reformen der Alterssicherung in Frankreich einen festen Bestandteil im nationalen Gesetzgebungsdiskurs. Dabei zeigt sich eine weitgehende Kongruenz der Reformpolitiken mit den Zielen der OMK/R, die Anwesenheit einer Vielzahl von Referenzen zur europäischen Dimension einschließlich des Koordinierungsprozesses und das Vorhandensein alternativer Erklärungsfaktoren auf der nationalen Ebene. Damit kann von einem katalysierenden Effekt der OMK/R auf die französischen Reformpolitiken in der Alterssicherung ausgegangen werden. Dieser Katalyseeffekt fällt stark aus, da die alternativen Erklärungsfaktoren sich ihrerseits durch umfangreiche Referenzen zur europäischen Dimension und, konkreter, zur OMK/R hervortun.

So verfügen die politischen Akteure in Frankreich über umfassende Informationen und Vergleichsstudien der europäischen Alterssicherungssysteme und der in den Nachbarländern durchgeführten Reformanstrengungen. Dabei wird die relative Position Frankreichs deutlich sichtbar. Dass sie bei vielen Einzelindikatoren Anlass zur Sorge gibt, nutzt die Regierung Raffarin im Jahr 2003, um das der offenen Politikkoordinierung inhärente Spiel des „naming, shaming and blaming ${ }^{\text {“3 }}$ gegen das eigene Land anzuwenden. Damit gelingt es ihr nachweislich, einzelne Reformmaßnahmen im parlamentarischen Gesetzgebungsprozess gegen Angriffe der Opposition zu immunisieren (Hacker 2010, S. 297ff.). Kein politischer Akteur möchte sich schließlich dem Bestreben des eigenen Landes in den Weg stellen, zur Gruppe der besten Schüler innerhalb der EU zu gehören.

Die Taktik ist besonders auffällig durch die vom einstigen Sozial- und Arbeitsminister Fillon über die vorliegenden Vergleichsstudien hinaus erfolgreich in die politische Arena transportierte Idee einer Rangordnung der in der EU durchgeführten Rentenreformen, ihrer Einzelbestandteile und ihrer Effekte. Das Bild vom einzigen Reformverweigerer Frankreich stimmt zwar nur bedingt mit der Realität überein (vgl. etwa COR 2001, S. 182ff.), ließ sich aber erfolgreich im parlamentarischen Diskurs implementieren. Denn auch bei den beiden Folgereformen zieht sich der „rétard français“ durch den Diskurs und findet seinen besonderen Ausdruck in der seit Jahren geringen Erwerbstätigkeit älterer Arbeitnehmer und im relativ geringen durchschnittlichen effektiven Rentenalter der Franzosen. Damit ist die Grundidee der OMK/R längst in der französischen Politik angekommen und dies auf eine interessante Art und Weise. Während etwa Deutschland die Tendenz zeigt, sich im Rahmen der OMK/R als übereifriger Schüler zu präsentieren, der unabhängig vom Koordinierungsverfahren bereits alle Zielkriterien erfüllt, sodass Kommission und Rat nicht den leisesten Kritikpunkt äußern können (vgl. etwa das Lob für die deutschen Rentenreformen in: Europäische Kommission 2009, S. 59), zeigt sich die französische Regierung in ihren Nationalen Strategieberichten offen für Veränderungsvorschläge und verschweigt nicht die Unzulänglichkeit des aktuellen Stands und der projizierten Entwicklungen ihres Alterssicherungssystems. Die hiermit programmierte Schelte im Rahmen der Gemeinsamen Berichte flankiert so den auf nationaler Ebene längst festgestellten Reformbedarf.

Die Analyse bestätigt die Annahme eines Einflusses der Koordinierungsmethode auf die französische Alterssicherungspolitik. Die politischen Akteure bedienen sich der OMK/R zur Flankierung ihrer eigenen Reformpläne, die wiederum in einer Art selbstständig entwickelten OMK/R auf bilateraler Basis vorbereitet wurden (Natali 2008, S. 231f.). Der Behauptung, dass der Effekt der OMK/R nur als marginal bezeichnet werden kann (Starzec 2009, S. 176), muss jedoch widersprochen werden. Vielmehr begleitet und verbindet sich die OMK/R mit den bereits im nationalen Zusammenhang stark europäisch diskutierten Reformbestrebungen und übt somit eine starke „Hebelwirkung“"bzw. einen stark katalysierenden Effekt aus (vgl. auch Erhel et al. 2005, S. 229).

2 So stehe stets die finanzielle Nachhaltigkeit de Rentensysteme im Zentrum der Gemeinsamen Berichte von Kommission und Rat, doch werde kaum über die Gewährleistung eines hohen Einkommensniveaus der Rentner gegenüber den aktiv Erwerbstätigen berichtet.

3 Dies bezeichnet den Versuch der Steuerung bzw. Disziplinierung politischer Prozesse über die Veröffentlichung von misslichen Zuständen und Nennung der Verantwortlichen. 


\section{Fazit}

Das Beispiel Frankreich lässt daran zweifeln, ob die OMK im Bereich der Altersrenten als „new mode of governance " gelten kann, die regulative oder gar redistributive Sozialpolitik besser macht, als dies die Mitgliedstaaten könnten (Lodge 2007). Ihr Wirkungsgrad ist nicht als lenkend einzustufen, und so zeigt sie auch keine Befähigung, die soziale Dimension der EU maßgeblich voranzutreiben. Interessant ist jedoch das ihr inhärente Potenzial, bestimmte Reformtendenzen auf nationaler Ebene zu stützen. Die OMK ist damit mehr als ein Forum zum Austausch von Informationen (Eckardt 2005; Eichenhofer 2009). In Frankreich vermochten es die Reformbefürworter der Regierung und ihrer parlamentarischen Mehrheit, die Ergebnisse des Koordinierungsverfahrens in ihre politische Strategie einzubinden. Bei den betrachteten Reformvorhaben standen fiskalpolitische Aspekte im Zentrum des Diskurses. Die als Mittel zur Stärkung des sozialen Charakters der EU eingeführte OMK dient durch ihre Nutzung als Beweismittel für die Notwendigkeit der vorgelegten Reformpläne somit der Befestigung und Katalyse des marktschaffenden Charakters der Integration.

Diese Entwicklung wird befeuert durch das Unvermögen der Reformgegner in Oppositionsparteien und Gewerkschaften, die $\mathrm{OMK} / \mathrm{R}$ als unterstützendes Vehikel in ihre eigenen Strategien zu integrieren. Die Vielfalt der Ziele und Indikatoren der OMK böte durchaus Optionen zur Thematisierung und Untermauerung alternativer Reformkonzepte, in deren Zentrum nachhaltige Alterssicherungssysteme und ein angemessenes Rentenniveau stehen (vgl. das Beispiel Belgien bei Vanhercke 2007).

Für die nun begonnene neue Zehnjahresstrategie der EU „Europa 2020“ sind all jene aufgefordert, die sich um die Angemessenheit der Rentenbezüge und den sozialen Zusammenhalt sorgen, das zu erkennende Potenzial der OMK als Kata- lysator ausgewählter Rentenpolitiken zu bedenken und zu nutzen. Dies gilt umso mehr, als die Kommission mit dem Grünbuch Rente andeutet, künftig eine stärkere Rolle im Feld der Alterssicherungspolitiken übernehmen zu wollen. Die dort gestellte Suggestivfrage: „Sollte der politische Koordinierungsrahmen aufEU-Ebene gestärkt werden?" (Europäische Kommission 2010, S. 20), wird mit einem europäischen Mehrwert beantwortet, der sich einstelle, da sich die Herausforderungen für alle Mitgliedstaaten ähnelten und die Reformen der Alterssicherungssysteme mit den Erfordernissen des Stabilitäts- und Wachstumspakts sowie der „Europa 2020“-Strategie vereinbar sein müssten. Wenn anstehende Rentenreformen jedoch weiterhin vor allem von Interessen der Haushaltskonsolidierung und der wirtschaftlichen Wettbewerbsfähigkeit geleitet sein sollten (vgl. Hacker/van Treeck 2010), wird die „wettbewerbsgetriebene Harmonisierung über Umwege" faktisch an die Stelle des Anspruchs treten, das Europäische Sozialmodell zu stärken.

\section{LITERATUR}

Assemblée Nationale (2003a): Compte rendu intégral des séances du mardi 21 janvier 2003, Nr. 4, vom 46e jour de séance,

in: Journaux Officiels

Assemblée Nationale (2003b): Compte rendu intégral des séances du jeudi 3 juillet, Nr. 73, vom 3e jour de séance de la session,

in: Journaux Officiels

Assemblée Nationale (2003c): Compte rendu intégral des seánces du mercredi 4 juin 2003, Nr. 52, vom 94e jour de séance de la session, in: Journaux Officiels

Assemblée Nationale (2003d): Compte rendu intégral. 3e séance du jeudi 12 juin, Nr. 57 (3), vom 240e séance, in: Journaux Officiels

Benz, A. (2007): Accountable Multilevel Governance by the Open Method of Coordination?, in: European Law Journal 13 (4), S. 505-522

Busch, K. (2009): Die europäischen Perspektiven des Wohlfahrtsstaats, in: Gawrich, A./Knelangen, W./Windwehr, J. (Hrsg.): Sozialer Staat - soziale Gesellschaft? Stand und Perspektiven deutscher und europäischer Wohlfahrtsstaatlichkeit, Leverkusen-Opladen/Farmington Hills, USA, S. 253-279

Chalmers, D./Lodge, M. (2003): The open method of co-ordination and the European welfare state, CARR discussion papers (11), hrsg. von der London School of Economics, London

Charpin, J.-M. (1999): L' avenir de nos retraites, Rapport au Premier ministre, Documentation Française, Paris

Citi, M./Rhodes, M. (2007): New Modes of Governance in the EU: Common Objectives versus National Preferences, European Governance Papers N-07-01
Commissariat général du Plan (1991): Livre blanc sur les retraites. Garantir dans l'équité les retraites de demain, unter Mitarbeit von Michel Rocard, Documentation Française, Paris

Conseil d'orientation des retraites (COR) (2001): Premier rapport.

Retraites: renouveler le contrat social entre les générations.

Orientations et débats, 06.12., Paris

Conseil d'orientation des retraites (COR) (2004): Retraites: les réformes en France et à l'étranger, le droit à l'information, Deuxième Rapport, 03.06., Paris

Conseil d'orientation des retraites (COR) (2006): Retraites: perspectives 2020 et 2050. Troisième rapport, 29.03., Paris Conseil d'orientation des retraites (COR) (2007): Retraites: questions et orientations pour 2008, Quatrième rapport, Adopté en janvier, Paris De la Porte, C. (2010): State of the art. Overview of concepts, indicators and methodologies used for analyzing the social OMC, RECWOWE Working-Paper 15, Edinburgh

De Ruiter, R. (2010): EU soft law and the functioning of representative democracy: the use of methods of open co-ordination by Dutch and British parliamentarians, in: Journal of European Public Policy 17 (6), S. $874-890$

Diaz-Bone, R. (2006): Zur Methodologisierung der Foucaultschen Diskursanalyse, in: Forum: Qualitative Social Research 7 (1), Art. 6, http.//nbn-resolving.de/urn:nbn:de:0114-fqs060168

Dolowitz, D. P./Marsh, D. (2000): Learning from abroad: The role of Policy Transfer in Contemporary Policy-Making, in: Governance 13 (1), S. 5-23 
Eckardt, M. (2005): The open method of coordination on pensions. An economic analysis of its effects on pension reforms,

in: Journal of European social policy 15 (3), S. 247-267

Eichenhofer, E. (2009): Offene Methode der Koordinierung und Alterssicherung, in: Deutsche Rentenversicherung 64 (1), S. 3-14 Erhel, C./Manin, L./Palier, B. (2005): The Leverage Effect. The Open Method of Co-ordination in France, in: Zeitlin, J./Pochet, P./Magnusson, L. (Hrsg.): The open method of coordination in action. The European employment and social inclusion strategies, Brussels, S. 217-247 Europäische Kommission (2003): Angemessene und nachhaltige Renten. Gemeinsamer Bericht der Kommission und des Rates, hrsg. vom Amt für Amtliche Veröffentlichungen der Europäischen Gemeinschaften, Luxemburg

Europäische Kommission (2006): Adequate and sustainable pensions. Synthesis report 2006, hrsg. vom Office for Official Publications of the European Communities, Luxembourg

Europäische Kommission (2009): Joint Report on Social Protection and Social Inclusion 2009, Country Profiles, 24.02., Brussels

Europäische Kommission (2010): Grünbuch. Angemessene, nachhaltige und sichere europäische Pensions- und Rentensysteme, KOM(2010)365 endgültig, vom 07.07.

Evans, M./Davis, D. (1999): Understanding Policy Transfer: A MultiLevel, Multi-Disciplinary Perspective, in: Public Adminstration 77 (2), S. $361-385$

Gouvernement de la République Française (2002): Rapport de Stratégie Nationale sur les Pensions, vom 11.09., Paris

Gouvernement de la République Française (2005): Rapport de Stratégie Nationale sur les Pensions, vom 15.07., Paris

Gouvernement de la République Française (2008): Rapport sur les stratégies nationales pour la protection sociale et l'inclusion sociale 2008-2010, vom 22.09., Paris

Hacker, B. (2010): Das liberale Europäische Sozialmodell. Rentenreformen in der EU und die Offene Methode der Koordinierung, Baden-Baden Hacker, B./van Treeck, T. (2010): Wie einflussreich wird die europäische Governance? Reformierter Stabilitäts- und Wachstumspakt, Europa 2020-Strategie und "Europäisches Semester", hrsg. von der Friedrich-Ebert-Stiftung, Berlin

Hartlapp, M. (2006): Über Politiklernen lernen. Überlegungen zur Europäischen Beschäftigungsstrategie, WZB Discussion Paper 2006-114, Berlin

Hodson, D./Maher, I. (2001): The Open Method as a New Mode of Governance: The Case of Soft Economic Policy Co-ordination, in: Journal of Common Market Studies (JCMS) 39 (4), S. 719-746 Holzinger, K./Jörgens, H./Knill, C. (Hrsg.) (2007): Transfer, Diffusion und Konvergenz von Politiken, Wiesbaden

Höpner, M./Schäfer, A. (2010): Grenzen der Integration - wie die Intensivierung der Wirtschaftsintegration zur Gefahr für die politische Integration wird, in: integration 33 (1), S. 3-20

Keller, R. (2007): Diskursforschung. Eine Einführung für SozialwissenschaftlerInnen, Wiesbaden

Leiber, S./Schäfer, A. (2008): Der doppelte Voluntarismus in der EU-Sozial- und Beschäftigungspolitik, in: Tömmel, I. (Hrsg.): Die Europäische Union. Governance und Policy-Making, S. 116-135
Lodge, M. (2007): Comparing Non-Hierarchical Governance in Action: the Open Method of Co-ordination in Pensions and Information Society, in: Journal of Common Market Studies (JCMS) 45 (2), S. 343-365 Natali, D. (2008): Pensions in Europe, European pensions. The evolution of pension policy at national and supranational level, Brussels Palier, B. (2003): La réforme des retraites, Que sais-je ? (3667), hrsg. von Presses universitaires de France, Paris

Platzer, H.-W. (2009): Europäisches Sozialmodell und sozialpolitisches Regieren (in) der EU. Zum integrationspolitischen Kontext und den Perspektiven der Offenen Methode der Koordinierung, in: Devetzi, S./ Platzer, H.-W. (Hrsg.): Offene Methode der Koordinierung und europäisches Sozialmodell. Interdisziplinäre Perspektiven, Stuttgart, S. 83-120 Radaelli, C.M. (2003): The Open Method of Coordination: A new governance architecture for the European Union?, Stockholm Rat der Europäischen Union (2006): Gemeinsame Stellungnahme des Ausschusses für Sozialschutz und des Ausschusses für Wirtschaftspolitik zur Mitteilung der Kommission: „Zusammen arbeiten, zusammen mehr erreichen: ein neuer Rahmen für die offene Koordinierung der Sozialschutzpolitik und der Eingliederungspolitik in der Europäischen Union“", 6801/06, vom 27.02., Brüssel

Scharpf, F. W. (1999): Regieren in Europa. Effektiv und demokratisch? Frankfurt a.M.

Schludi, M. (2005): The reform of Bismarckian pension systems. A comparison of pension politics in Austria, France, Germany, Italy and Sweden, Amsterdam

Sénat (2003): Commission des finances, du contrôle budgétaire et des comptes économiques de la Nation: Avis sur le projet de loi portant réforme des retraites, par $M$. Adrien Gouteyron (383), vom 07.07., Paris Sénat (2007): Compte rendu intégral. Séance du mardi 2 octobre (46), vom 2e jour de séance de la session, Paris

Sénat (2008a): Compte rendu intégral, Séance du mercredi 12 novembre (89), vom 20e jour de séance de la session, in: Journaux Officiels, Paris

Sénat (2008b): Compte rendu intégral. Séance du mardi 18 novembre (92), vom 23e jour de séance de la session, in: Journaux Officiels, Paris Starzec, C. (2009): France's national model of the welfare state: tradition and changes, in: Golinowska, St./Hengstenberg, P./Zukowski, M. (Hrsg.): Diversity and commonality in European social policies. The forging of a European social model, Wydawnictwo Naukowe Scholar (Study 2008), Warsaw, S. 140-183

Urban, H-J. (2003): Europäisierung der Gesundheitspolitik? Zur Evolution eines Politikfeldes im europäischen Mehrebenen-System, Papers der Forschungsgruppe Public Health SP I 2003-303, hrsg. vom Wissenschaftszentrum Berlin für Sozialforschung (WZB), Berlin

Vanhercke, B. (2007): The operation of the social protection and social inclusion OMC in Belgium: A hybrid policy instrument between hard and soft law, Paper for the EUSA tenth biennial international conference 17-19 May, Montreal

ZohInhöfer, R./Ostheim, T. (2007): Politiktransfer durch die Europäische Beschäftigungspolitik? Methodische Überlegungen und empirische Analyse des Falls Deutschland, in: Holzinger, K./Jörgens, H./Knill, C. (Hrsg.): Transfer, Diffusion und Konvergenz von Politikern, Wiesbaden, S. $327-347$ 